\title{
Non-invasive electro-gastro-intestinogram (EGIG) recording under physiological conditions
}

\author{
FEKETE László ${ }^{1}$, BAKITY Boldizsár², MICSKÓ Anna³, BARANYÁK \\ Zsuzsanna ${ }^{4}$, BÁRDOS György ${ }^{5}$
}

\begin{abstract}
Electro-intestinogram (EIG), together with electro-gastrogram (EGG), are non- invasive methods by which gastro-intestinal (GI) activity can be monitored contin- uously and without disturbing the patient. They also make it possible to determine whether disturbed functioning require any acute or planned intervention, and also to assess results of earlier (pharmacological or surgical) treatments.

Based on scarce literary sources and on our own experimental experiences, a new device has been developed for non-invasive recording of the electrical activity of the whole gastro-intestinal system in a continuous way. Results of our preliminary testing revealed that the dominant frequency of the stomach is 1-5 cpm, of the small intestine 10-14 cpm, and of the large intestine 1-7 cpm, respectively. It has also been shown that changes of GI activity could be monitored by the device. Keywords: electro-intestinogram, non-invasive recording, gastro-intestinal activity
\end{abstract}

\section{Background}

The structure of different portions of the gastro-intestinal (GI) system show remarkable sim- ilarities, although differences also exist. The wall of the hollow organs usually contains 2-3 layers of smooth muscle, a circular, a longitudinal and in some places a longitudinal/diago- nal. The inner surface is always covered by a mucosal while the outer surface by a serosal layer. The latter is closely associated with the mesenteria that host blood vessels and nerves supplying the GI organs. [1]

Regulation of the GI system is at least threefold: intrinsic nervous, autonomic nervous and hormonal/paracrine. The intrinsic regulatory system consists of 4 plexi, of which the two larger — the myenteric (Auerbach) and the submucosus (Meissner) plexus represent the main structures. These plexi consist of a relatively large and rich neuronal network resem- bling that of some brain structures (hence they are frequently called "visceral brain") and reg- ulate peristalsis as well as other motility activities. In addition, this intrinsic neural network regulates local enzyme and digestive juice production as well. [1] [2] [3]

The extrinsic nervous control is represented by the GI branches of the autonomic nervous system. The basically noradrenergic sympathetic nerves usually inhibit motility and pro-

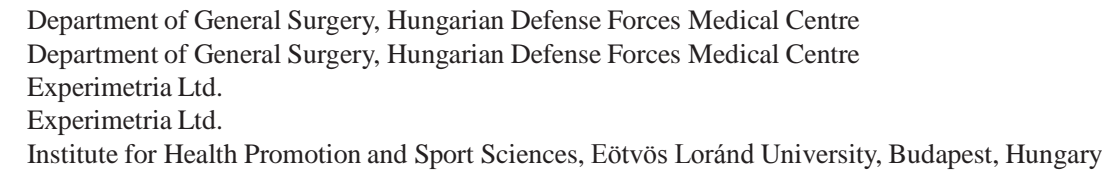


duction of digestive juices, whereas cholinergic dominance by the parasympathetic system enhances motility and enzyme production, respectively. The sympathetic fibers run from the spinal cord via the coeliac ganglia to the organs while the parasympathetic fibers run directly to the organs and form synapses in the GI wall. This way the extrinsic autonomic control modulates the activity of the intrinsic nervous system which in turn works in a reflexive way. The autonomic nervous system connects the visceral activity to the central nervous system, among others, to the spinal cord, medulla, limbic system and hypothalamus. [2]

GI activity is further modulated by hormones and paracrine transmitters produced mainly by endocrine glands within or closely associated with the GI tissue. These amino acid deriv- atives and neuropeptides act frequently locally as paracrine transmitters (e.g. gastrin in the stomach wall) and also as hormones (cholecystokinin, secretin, and gastrin itself) and repre- sent a feed-back/feed forward system within the gastro-intestinal structures.

In the complicated network of neuronal-hormonal regulation, the intrinsic plexi represent the final common pathway: all higher regulatory influences converge at these neurons and modulate local regulation. Although enzyme and juice production is an important feature of the operation of the GI system, motility is usually regarded as the factor representing normal activity of the stomach and the intestinal system. The myoelectric signals generated within the GI organs determine motility hence monitoring this activity may give an insight into the operation of the GI system. [4] So far, however, despite the long-known electro-gastrogram recording, very few attempts have been made to record the myoelectric activity of the whole GI system continuously and simultaneously and to get a general and detailed impression of the activity of the whole system.

Monitoring the activity of the whole GI system seems to be important for judging the intactness of the system for the gastroenterologist, and even more for the GI-surgeon follow-ing a surgical intervention. Non-invasive methods should be preferred in this respect since in this way the physician may get information without really disturbing the patient. A special application of the non-invasive GI activity monitoring using surface electrodes and mobile equipment is on the battle-field where the physician has a very short interval (the so called "golden hour") to determine whether the wound of the soldier requires immediate interven- tion and hence a priority for transport to the hospital or a few hours may be allowed until the wounded person gets further medical help.

EGG activity seems to well correlate with gastric emptying in healthy subjects if tested with a solid test meal. [5] [7] It has also been shown that cutaneous EGG shows consequent frequency and power increase if tested with a solid meal, whereas it shows inconsistent re- sponse (power increase but frequency decrease) when tested by a large water load. [6] EGG measures seem to be fairly reproducible and relatively stable even over days or weeks. [8] Postprandial EGG may also reflect abnormal gastric functioning, like recurrent nausea and vomiting, by an increased instability, by decreased second harmonics and by tachygastria. [9] To sum it up, gastric electrography has proven to be a useful tool in the hands of gastroenter- ologists since it provides a non-invasive way by which gastric functions and malfunctions could be monitored. [4]

Despite the positive results on the EGG, due probably to technical difficulties, this meth- od has not gained much recognition in practice. It is also mysterious why electrographic methods have not been used to monitor intestinal motility. The few such studies (e.g. Brown et al., 1975) revealed that significant differences exist among the different parts of the gas- 
tro-intestinal system, regarding dominant frequency, which could offer an excellent tool to get information on the operation of the whole system. [10]

The aim of this paper, hence, was to describe a new device and method for non-invasive monitoring of the myoelectric activity of the whole GI system, continuously and simulta- neously, by combining the electro-gastrogram (EGG) with the electro-intestinogram (EIG) in one recording. As far as the authors know, up to now there has not been any diagnostic procedure that may follow the functional changes of the whole GI system and could provide valuable data on-line. [11]

\section{Methods}

\section{Equipment}

The electric signals of the nanovolt range were properly filtered to remove the influence of the breathing and circulatory activity and to save only the GI signals. A special extracellular amplifier and special software have been developed to process the incoming electric signals (SPEL Advanced ISOSYS System, Experimetria Ltd, Budapest, Hungary). Most of the nec- essary processing has been transferred from the electronic device to the software, including filtering. Figure 1 shows screen shots of a record made during the validation process (see below) with different filter settings, whereas Figure 2 shows power spectra created form this record also with different filter settings (bottom channel). In this study, a bandpass filter was set at 1 to $14 \mathrm{cpm}$ during recording.

To process the signals, power spectra were created by different filter settings at the re- spective ranges of the different GI portions: 1-3 cpm for the stomach, 2-5 cpm for the colon, and 9-14 cpm for the small intestine. To compare individual records, the dominant peak frequency and the power and maximum magnitude of that peak were calculated for each part of the GI system. (See Figure 1 and Figure 2 for examples.)

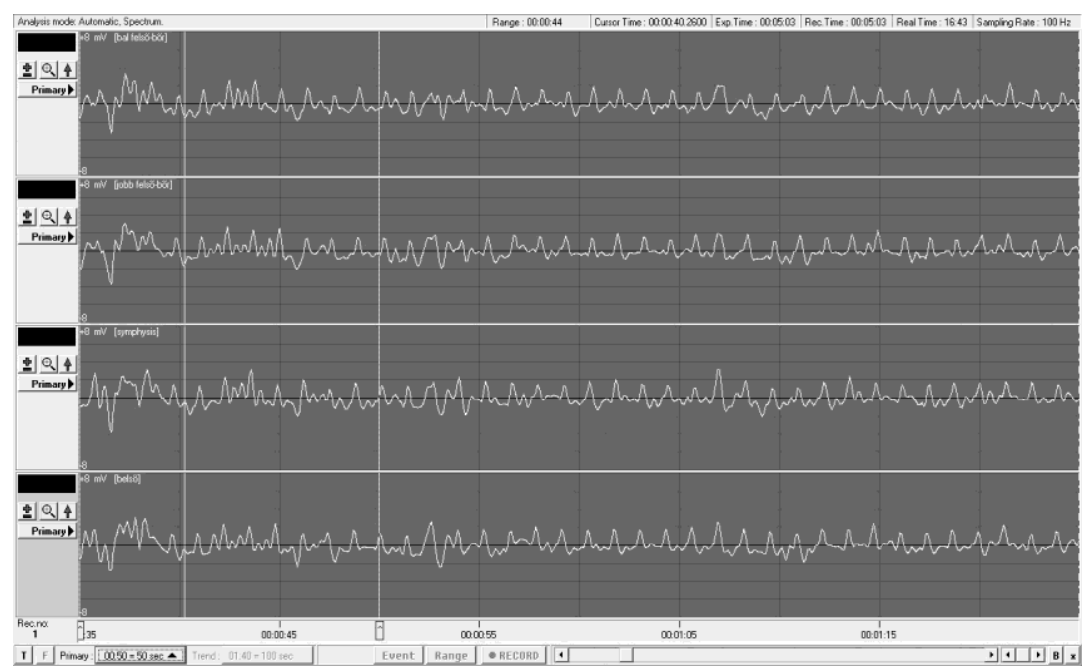

1/A. Unfiltered record. 
FEKETE L. et al.: Non-invasive electro-gastro-intestinogram (EGIG) recording...

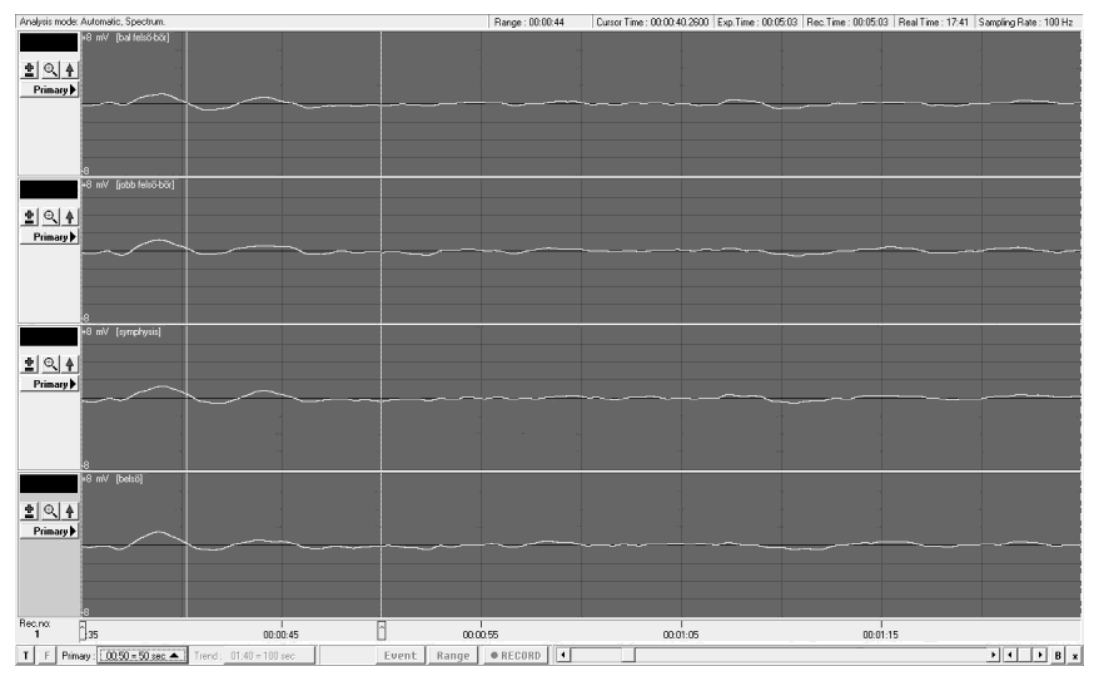

1/B. Bandpass filter set between 1 and $14 \mathrm{cpm}$ (full GI activity).

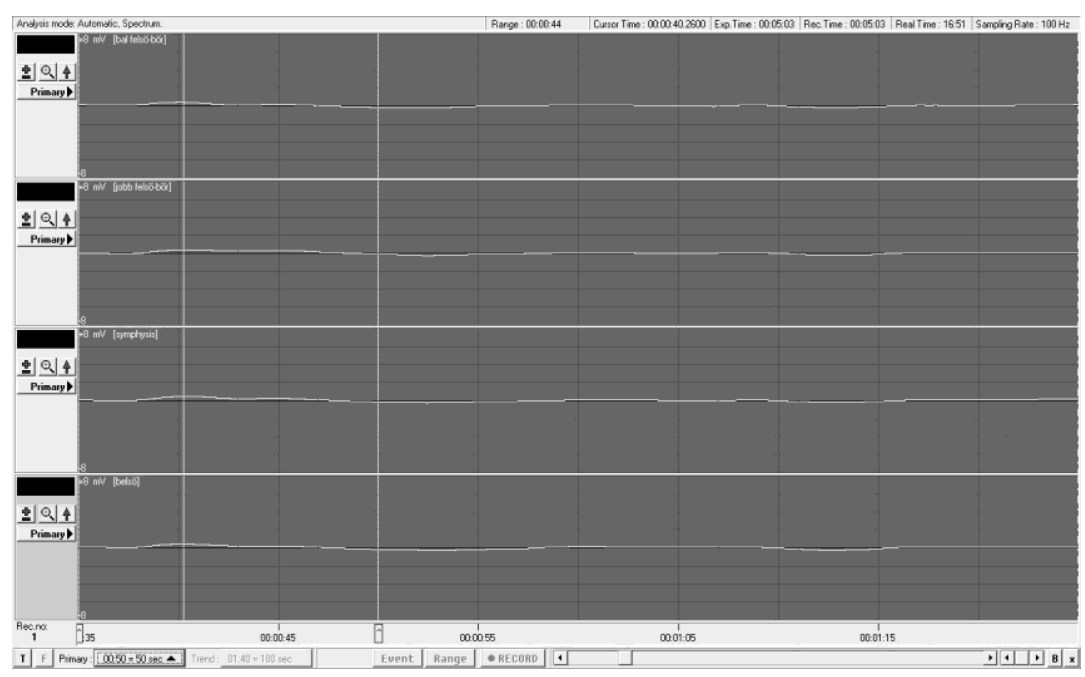

1/C. Bandpass filter set between 1 and 5 cpm (colon activity range).

Figure 1. Record screen obtained during the surgical procedure. Channel assignment from top to bottom: upper left skin electrode,

upper right skin electrode, symphysis electrode, colon-direct electrode. 


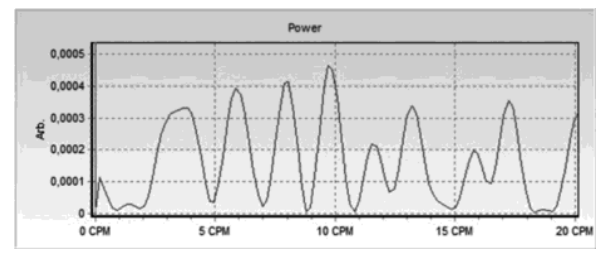

2/A. Unfiltered.

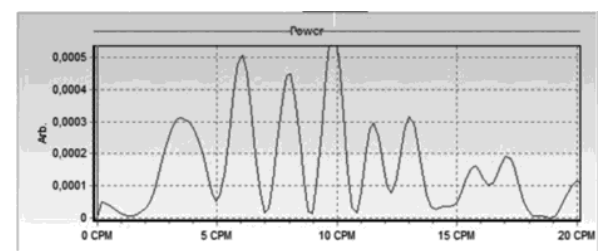

2/B. Bandpass filter set between 1 and $20 \mathrm{cpm}$ (full GI activity).

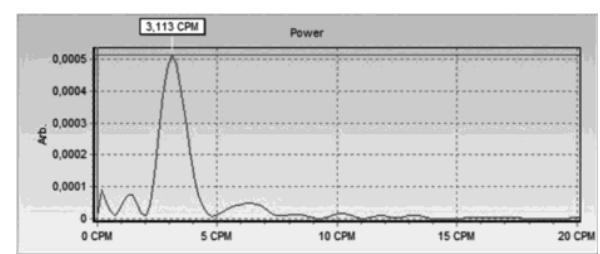

2/C. Bandpass filter set between 1 and $5 \mathrm{cpm}$ (colon activity range).

Figure 2. Power spectrum of Channel 4 (direct electrode on the colon) with different bandpass filter settings.

\section{Procedure}

Two experiments were run: one to validate the recording device and the measured signals, and another to demonstrate the capacity of the method in monitoring functional changes of the GI system.

\section{Validating the method}

32 subjects (11 males and 21 females, mean age 48.09 years) were tested. All subjects were prepared for a laparoscopic surgical intervention to remove the gall bladder. Subjects were intrathecally narcotised and were also given a muscle relaxant prior to the surgery. The lap-aroscopic intervention was made by keeping $12 \mathrm{mmHg}$ intra-abdominal pressure. Before removing the gall bladder, a pair of non-polarizing inert electrodes was implanted into one of different portions of the gastro-intestinal system, including the gastric corpus, the intraperi- toneal portion of the duodenum, the jejunum and the transversal colon, respectively. Simulta- 
neously, 3 pairs of electrodes were attached to the abdominal skin (upper left and upper right abdomen, and over the symphysis, respectively). 10 minutes before initiating the recording, the positive intra-abdominal pressure was released.

Bipolar recording was made parallel from the four pairs of electrodes during 5 minutes without making any intervention. Finally, the electrodes were removed and the surgical pro- cedure continued.

\section{Monitoring functional changes}

45 subjects (14 males and 31 females, mean age 20.04 years, age range 18-25 years, with negative laboratory tests and a normal abdominal ultrasound record) volunteered for the ex- periment. They were not given any money or other reward for the participation. Exclusion criteria were former GI surgery, cardio-vascular diseases, absorption and motility disorders, gastro-duodenal ulcer, and inflammatory bowel disease.

Four recording cup-electrodes were attached to the carefully cleaned abdominal skin, forming a square. A pair of them were placed $5 \mathrm{~cm}$ below the nipples and two of them

$1 \mathrm{~cm}$ below the navel, 10 and $15 \mathrm{~cm}$ apart the midline, respectively. (Picture 1 and Figure 3) A reference electrode was attached to the skin of the right thigh.

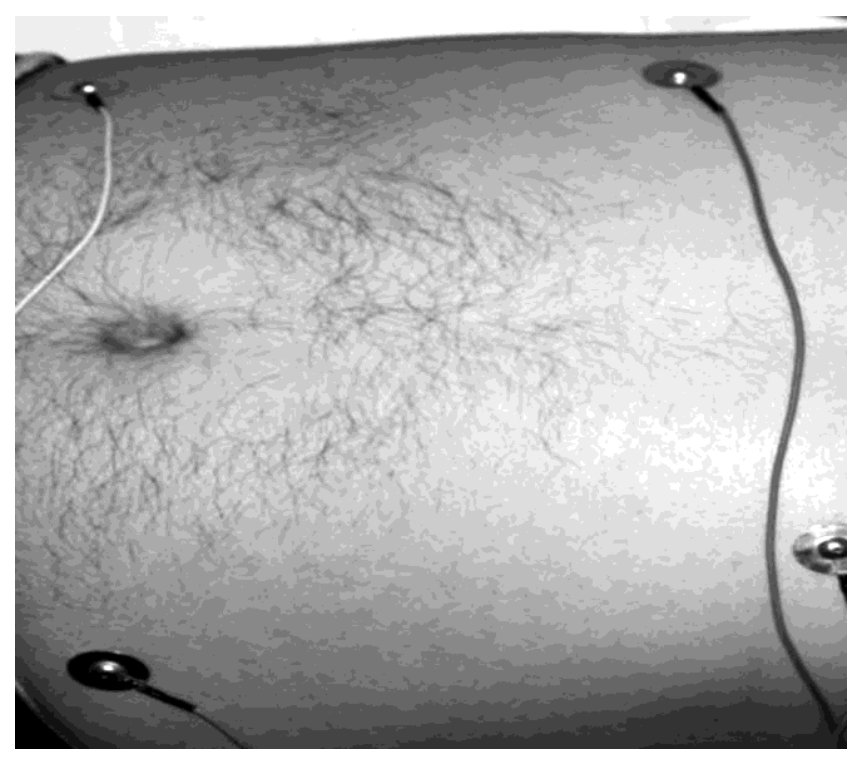

Picture 1. Electrode arrangement on the abdominal skin for recording GI activity (EGIG). 


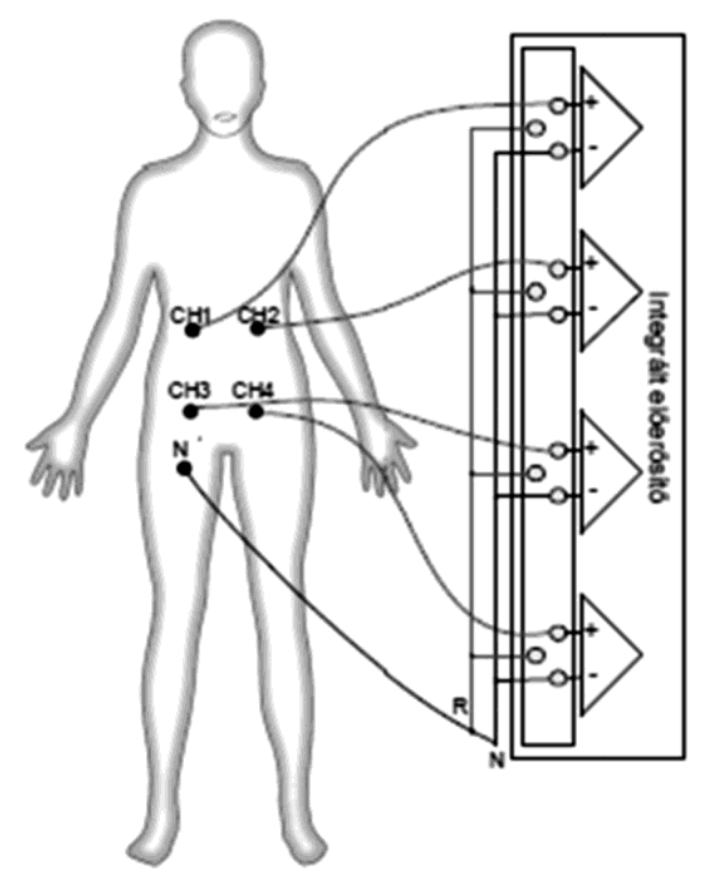

Figure 3. Sketch of the electrode connections to the recording device.

First, a 30 minute basal recording was made (preprandial phase) while the subjects were lying in a supine position without moving or talking. Then they were given 4 grams pro body- weight of $70 \%$ black chocolate to stimulate enteral CCK release. After a 5 minutes interval, another 30 minute recording was made (postprandial phase).

\section{Data processing}

Data were processed by the SPEL Advanced ISOSYS System (Experimetria Ltd, Budapest, Hungary). Results of the two periods were compared by a paired Student's t-test. A 0.05 significance level was kept throughout.

\section{Results}

\section{Validation of the method}

Records made during the surgical procedure clearly prove that the spectral distribution of the GI activity measured directly versus indirectly (superficially) have the same patterns, although, of course, the tissue signals have larger power than those recorded from the skin. Figure 4 shows power spectra of a record made with the internal electrode put on the colon. It is clear that all four electrodes record similar signals, with the symphysis electrode (i.e. the one closest to the colon) being almost identical with the colon-direct electrode and the two abdominal electrodes showing less power in the range of colonic activity. 
FEKETE L. et al.: Non-invasive electro-gastro-intestinogram (EGIG) recording...
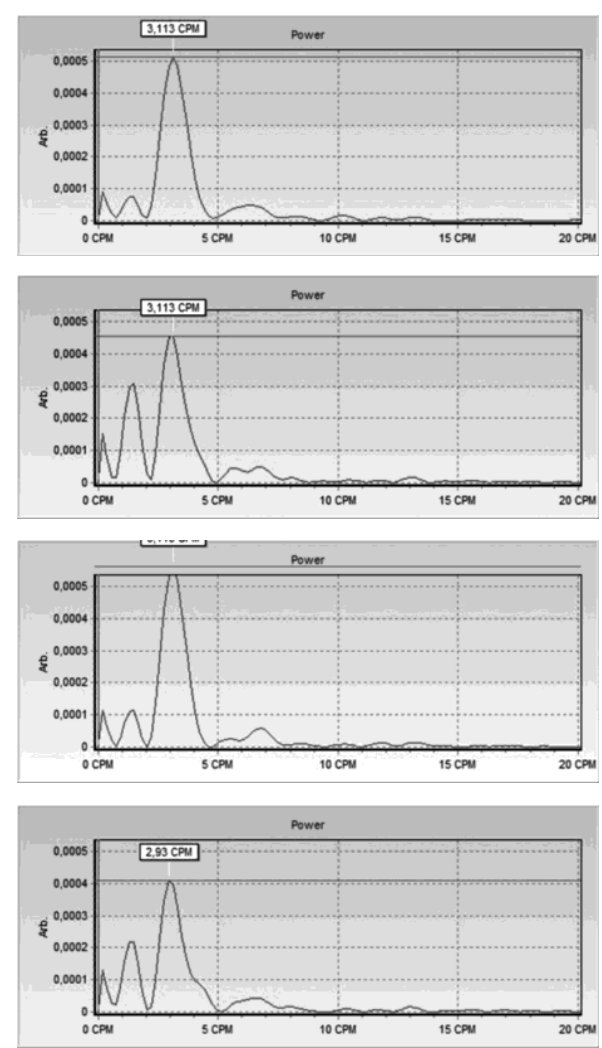

Figure 4. Comparison of the parallel records of the 4 electrodes made during the surgery.

Channel assignment from top to bottom and to right: upper left skin electrode, upper right skin electrode, symphysis electrode, colon-direct electrode.

Figure 5 displays spectra specifically filtered for the stomach, the jejunum and the colon, respectively. It was found that the different electrodes mirror the internal activity pretty well, with minor differences depending on the recording site. Stomach activity peaked around $2 \mathrm{cpm}$, small intestine activity between 9 to $13 \mathrm{cpm}$ and colon activity around 5 cpm, respec- tively. (Figure 6) 


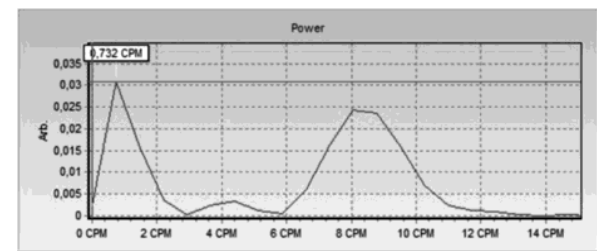

Electrode inside, on the stomach

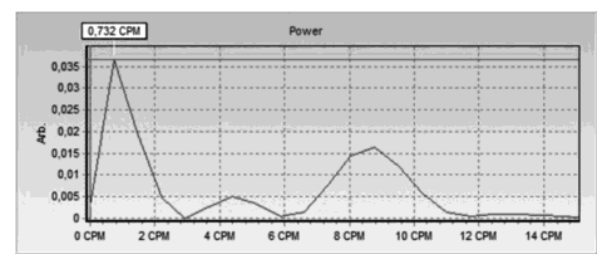

Electrode outside, on the right

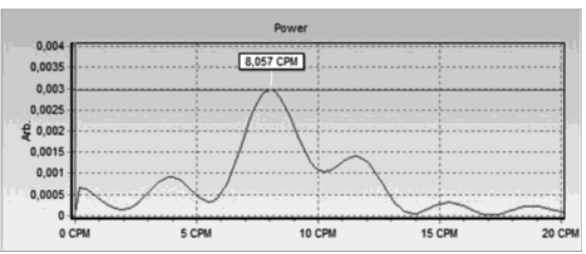

Electrode inside, on the jejunum

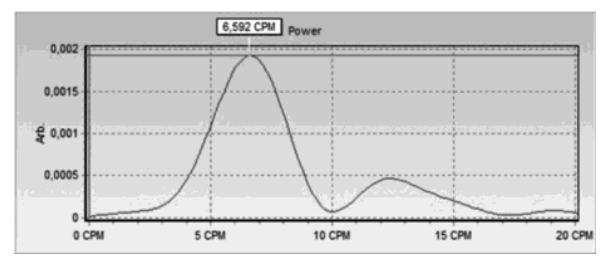

Electrode outside, on the right upper abdomen

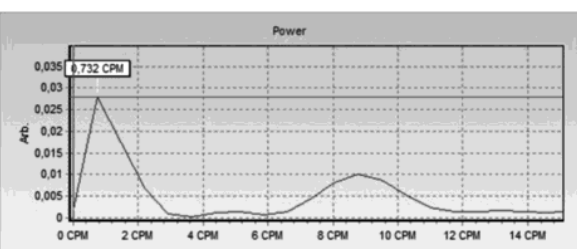

Electrode outside, close to the symphysis

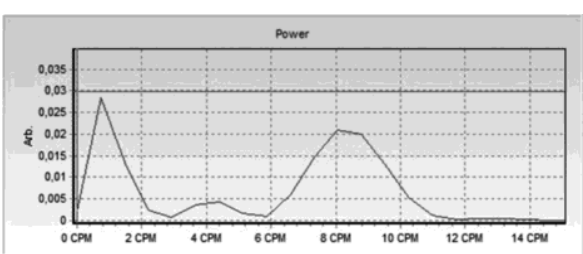

Electrode outside, on the left upper abdomen 5/A. Gastric activity.

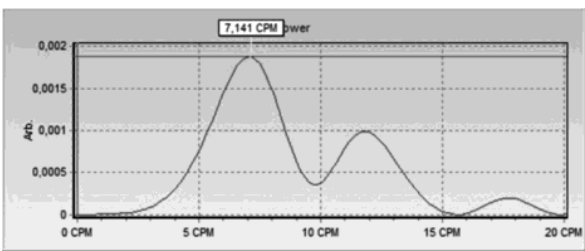

Electrode outside, close to the symphysis

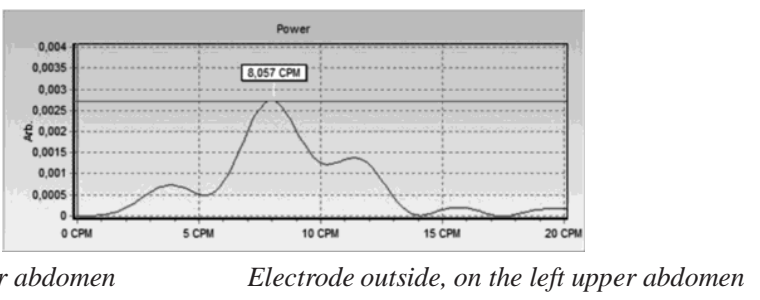

5/B. Small intestinal (jejunal) activity.

Figure 5. Power spectra of the gastrointestinal motility recorded during the surgery.

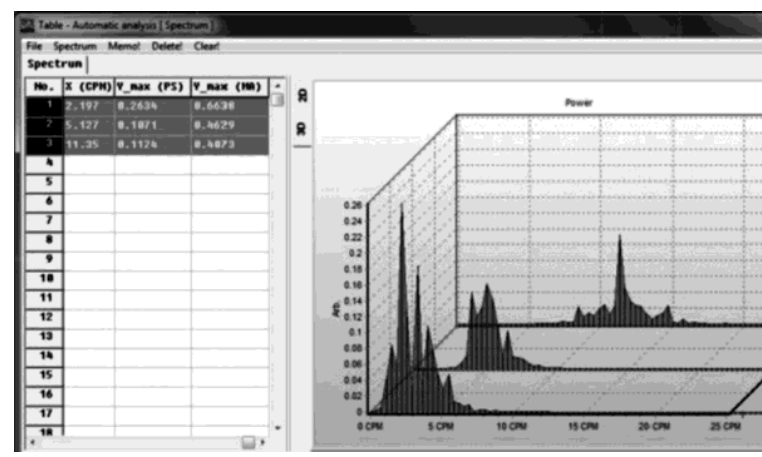

Figure 6. Comparison of the electric activity of the three portions of the GI system recorded by the combined EGIG method Power spectra from the front to the back: stomach, colon, small intestine. 


\section{Monitoring functional changes}

Figure 7 shows representative spectra of a subject while lying supine quiet, talking, hyper- ventilating and after consuming chocolate, respectively. The record, obtained from the right upper electrode, clearly shows that spectra are influenced by extra-gastrointestinal events like talking (Row A2) and hyperventilation (Row A3), but if the subject is quiet in a supine position (Row B1), the spectral changes clearly reflect the motor activity of the stomach and intestine, respectively. (Row A1 vs Row B2)

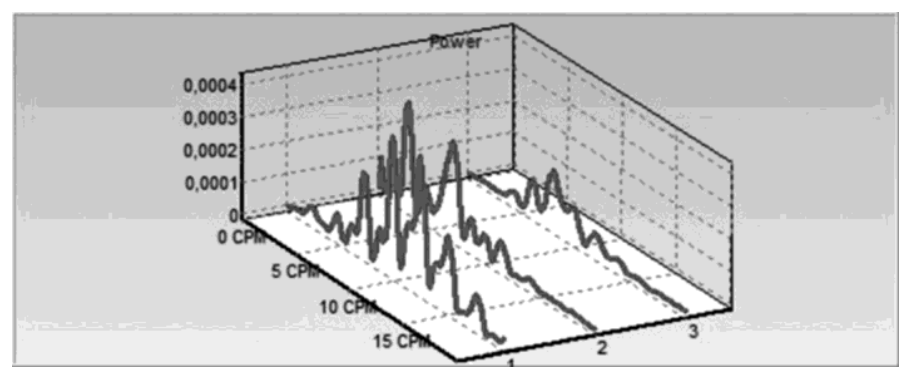

7/A.

Panel B. Row 1: Lying quiet and relaxed. Row 2: After consuming $4 \mathrm{~g} / \mathrm{bw}$ chocolate.

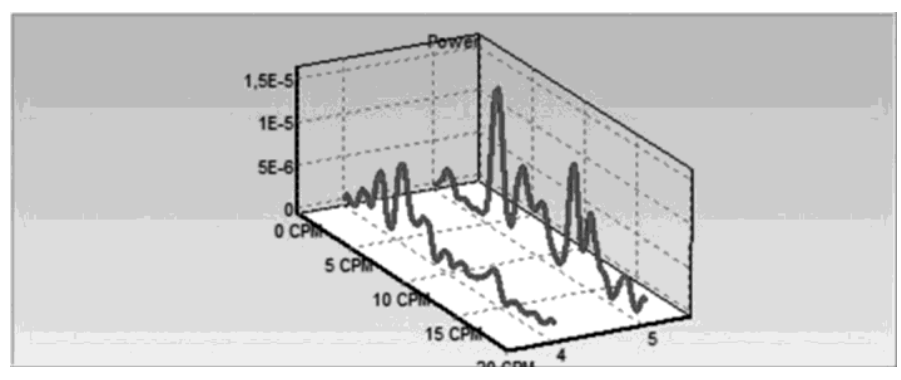

$7 / B$.

Panel A. Row 1: lying supine, quiet and motionless, hungry. Row 2: Subject talking (still lying supine). Row 3: Forced hyperventilation.

Figure 7. Power spectra of an individual in the chocolate feeding test.

Figure 8 shows the mean frequency differences between the hungry and chocolate fed states, respectively. Although the feeding had not elicited a major frequency shift in either of the studied GI sites, stomach (low frequency range, upper electrodes) and the intestines (middle frequency range, lower electrodes) behave in a different way: whereas in the stom- ach activity range we detected slight increases of frequency on the upper electrodes (i.e. those above the stomach) and a decrease on the lower electrodes (i.e. above the intestines), on the middle frequency range it is just the other way around. One may speculate that these changes reflect that the altered motor activity: mixing movements had been replaced by peristalsis. This interpretation is supported by the power and by the magnitude data which are summa- 
rized in Table 1 both power and the maximum magnitude decreased significantly in the low frequency ranges referring to the altered shape of the power spectrum.

As of the effect of chocolate consumption, the results are non-consequential (although the records exactly show all changes for each individual): about 30-40\% of the participants showed an increased and another 30-35\% a decrease in GI activity, the rest being essentially unchanged.

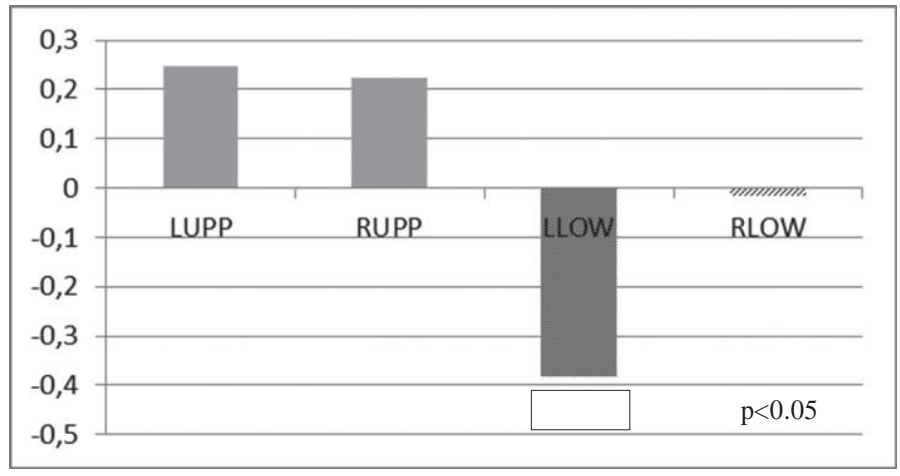

8/A. Low frequency range.

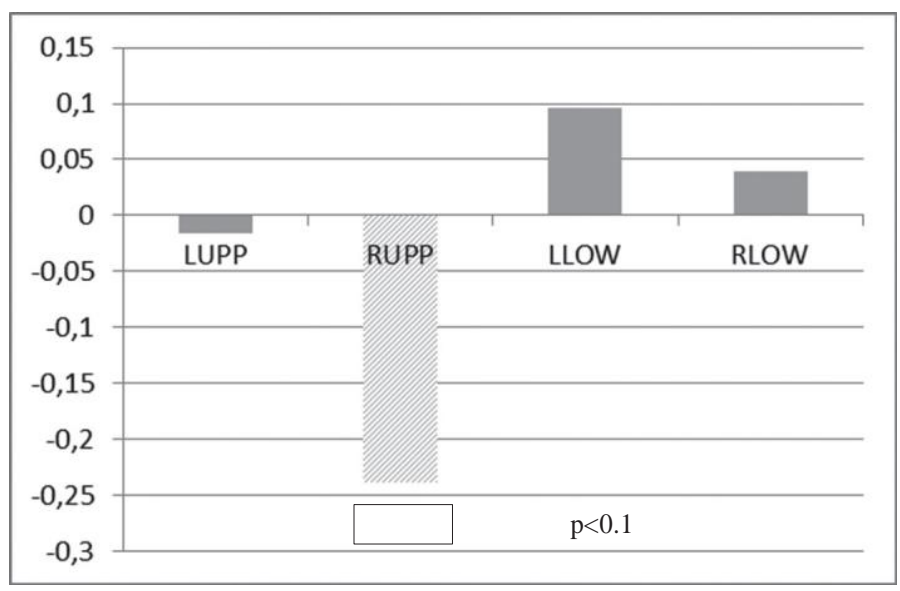

8/B. Middle frequency range.

Figure 8. Mean frequency differences between the hungry versus chocolate fed states.

LUPP: right upper electrode; RUPP: left upper electrode; LLOW: left lower electrode; RLOW: right lower electrode. 
Table 1. Mean power and magnitude differences, respectively, between the hungry versus chocolate fed states.

\begin{tabular}{|c|c|c|c|c|}
\hline \multicolumn{2}{|c|}{ POWER } & & & \\
\hline \multicolumn{5}{|c|}{ LOW FREQUENCY RANGE } \\
\hline \multicolumn{2}{|r|}{$\operatorname{MEAN}\left(* 10^{-3}\right)$} & $\pm \mathrm{SE}$ & t-value & $\mathrm{p}$ \\
\hline LUPP & $-28,2182$ & 0,017879 & $-1,596$ & $\mathrm{p}<0,1$ \\
\hline RUPP & $-40,9515$ & 0,0201146 & $-2,058$ & $\mathrm{p}<0,025$ \\
\hline LLOW & $-17,2167$ & 0,0105915 & $-1,643$ & $\mathrm{p}<0.05$ \\
\hline \multirow[t]{3}{*}{ RLOW } & $-26,3850$ & 0,017766 & $-1,502$ & $\mathrm{p}<0,1$ \\
\hline & \multicolumn{3}{|c|}{ MIDDLE FREQUENCY RANGE } & \\
\hline & MEAN & $\pm \mathrm{SE}$ & t-value & $\mathrm{p}$ \\
\hline LUPP & 0,2240 & 0,0002346 & 0,9653 & ns \\
\hline RUPP & 0,2240 & 0,0000957 & 0,7152 & ns \\
\hline LLOW & $-0,2565$ & 0,0002023 & $-1,282$ & ns \\
\hline RLOW & 0,0378 & 0,0001379 & 0,2771 & ns \\
\hline \multicolumn{5}{|c|}{ MAGNITUDE } \\
\hline \multicolumn{3}{|c|}{ LOW FREQUENCY RANGE } & & \\
\hline \multicolumn{2}{|r|}{$\operatorname{MEAN}\left(* 10^{-3}\right)$} & $\pm \mathrm{SE}$ & t-value & $\mathrm{p}$ \\
\hline LUPP & $-41,6002$ & 0,0185457 & $-2,268$ & $\mathrm{p}<0,001$ \\
\hline RUPP & $-60,6860$ & 0,0213079 & $-2,88$ & $\mathrm{p}<0,001$ \\
\hline LLOW & $-18,4661$ & 0,0155884 & $-1,198$ & $\mathrm{p}<0.05$ \\
\hline \multirow[t]{3}{*}{ RLOW } & $-\mathbf{3 3}, \mathbf{5 8 3 3}$ & 0,0187723 & $-1,809$ & $\mathrm{p}<0.05$ \\
\hline & \multicolumn{3}{|c|}{ MIDDLE FREQUENCY RANGE } & \\
\hline & MEAN & $\pm \mathrm{SE}$ & t-value & $\mathrm{p}$ \\
\hline LUPP & 1,0835 & 0,0029376 & 0,3729 & ns \\
\hline RUPP & $-0,4395$ & 0,0016904 & $-0,263$ & ns \\
\hline LLOW & $-3,8401$ & 0,0029461 & $-1,318$ & $\mathrm{p}<0,1$ \\
\hline RLOW & $-0,1019$ & 0,0022887 & $-0,045$ & ns \\
\hline
\end{tabular}

LUPP: right upper electrode; RUPP: left upper electrode; LLOW: left lower electrode; RLOW: right lower electrode.

\section{Discussion}

The new device and software provide a non-invasive method by which the activity of the gastro-intestinal system can be monitored continuously and without much disturbing of the subject or patient. The electro-gastro-intestinogram (EGIG), obtained through superficial 
FEKETE L. et al.: Non-invasive electro-gastro-intestinogram (EGIG) recording...

extra-abdominal electrodes may be a novel instrumental aid for surgical practice. This non- invasive method might make it possible to detect dysfunctional intestinal activity and espe- cially those with decreased or stopped motor function in a very short time without placing the patient into complicated equipment, and also to decide whether an instant intervention was necessary. Due to the small size of the device and to the portable (off power) computer technology, an instant recording could also be made on the field, even under battle conditions hence providing a useful on-site tool for military doctors.

In addition, this method might be used to examine extreme states in which the visceral organs suffer big load and/or which amount to unusual stress, such as military pilots have to take when flying with a load of even 10G. Up to now, we do not know too much about the viscero-motor changes under and following flying conditions, thus the method described now may open new insight into this problem.

\section{References}

[1] GANONG, W. F.: Review of Medical Physiology. Appleton and Lange - Prentice-Hall Internat. Inc., 1989.

[2] LOEWY, A. D., SPYER, K. M. (Eds.): Regulation of Autonomic Functions. Oxford-New York: Oxford University Press, 1990.

[3] NILSSON, S., HOLMGREN, S. (Eds.): Comparative Physiology and Evolution of the Autonomic Nervous System. Chur: Harwood Academic Publishers, 1994.

[4] STERN, R. M., KOCH, K. L. Electrogastrography. Santa Barbara: Praeger, 1985.

[5] BUREŠ, J., KOPÁČOVÁ, M., VOŘÍŠEK, V., BUKAČ, J., NEUMANN, D., ŽIVNÝ, P., PALIČKA, V., REJCHRT. S.: Correlation of electrogastrography and gastric emptying rate estimated by $13 \mathrm{C}$-octanoic acid breath test in healthy volunteers. Folia Gastroenterol Hepatol, 51 (2007), 5-11.

[6] CHEN, J. D, Z., McCALLUM, R. W.: Response of the electrical activity of the human stomach to water and a solid meal. Medical \& Biological Engineering \& Computing, 29 (1991), 351-7. DOI: https:// doi.org/10.1007/BF02441654

[7] CHEN, J. D. Z., McCALLUM, R. W., RICHARDS, R.: Frequency components of the electrogastrogram and their correlations with gastrointestinal contractions in humans. Medical \& Biological Engineering \& Computing, 31 (1993), 60. DOI: https://doi.org/10.1007/BF02446895

[8] JONDERKO, K., KASICKA-JONDERKO, A., KRUSIEC-SWIDERGOL, B. (et al.): How reproducible is cutaneous electrogastrography? An in-depth evidence-based study. Neurogastroenterol Motil, 176 (2005), 800-9. DOI: https://doi.org/10.1111/j.1365-2982.2005.00707.x

[9] GELDOF, H., van der SCHEE, E. J., van BLANKENSTEIN, M., GRASHUIS, J. L.: Electrogastrographic study of gastric myoelectrical activity in patients with unexplained nausea and vomiting. Gut, 27 (1986), 799-808. DOI: https://doi.org/10.1136/gut.27.7.799

[10] BROWN, B. H., SMALLWOOD, R. H., DUTHIE, H. L., STODDARD, C. J.: Intestinal smooth muscle electrical potentials recorded from surface electrodes. Medical \& Biological Engineering \& Computing, 13 (1975), 97-103. DOI: https://doi.org/10.1007/BF02478194

[11] SIMONIAN, H. P., PANGANAMAMULA, K., PARKMAN, H. P. (et al.): Multichannel electrogastrography (EGG) in normal subjects: a multicenter study. Digestive Diseases and Sciences, 49 (2004), 594-601. DOI: https://doi.org/10.1023/B:DDAS.0000026304.83214.50 